\title{
Modeling the Nonlinear Dynamics of Nanotube Cores Driven by Interlayer Dispersion Force Modulation: New Developments and Future Applications
}

\author{
F. PINTO* \\ Jazan University, Faculty of Science, Department of Physics 45142 Gizan, Saudi Arabia
}

\begin{abstract}
Despite several past proposals to employ the inner cores of multiwalled nanotubes as, among others, ultra-highfrequency oscillators, memory devices, and nano-scale sensors, driving into motion a mass initially at rest within the nanotube outer walls has remained a crippling practical obstacle. In addition to the challenge of applying an external driving force upon the entirely embedded shuttle, it has been reported that the dynamics of such motion is "truly nonlinear", that is, it cannot be reduced to that of harmonic or nearly-harmonic oscillators even in the case of vanishing amplitudes. The author has shown that, since friction is nearly negligible, the inner core can be set into motion by breaking the high axial symmetry of the interlayer dispersion forces exerted on it by the outer walls. For instance, by fabricating nanotubes with even just two segments having slightly different dielectric properties, it was concluded that the motion of a partially extruded core under the action of an external electric field could be remarkably stabilized and electrical energy could be both stored into and released from the van der Waals field. Further significant progress was made by identifying a possible mechanism for the time-modulation of the spectral properties of double-walled nanotubes by acting on the free-carrier exciton screening in semiconducting nanotubes. In this paper, new developments are presented in the accurate mathematical modeling of these complex driven systems and additional future applications of telescoping nanotubes as actuators, non-electrochemical energy nanostorage systems, and neutral particle accelerators are illustrated.
\end{abstract}

DOI: 10.12693/APhysPolA.129.819

PACS/topics: 07.20.Pe, 84.60.Ve, 85.85.+j, 42.50.Pq, 34.20.Cf

\section{Introduction}

The critical role of dispersion forces between atoms and surfaces in industry was already clear in the first part of the twentieth century [1]. Notably, reports of practical challenges faced by colloid scientists at the end of the 1920s provide a firm impression of knowledge gained from continued exposure to industry and of experimental activity acutely motivated by interest in technological applications [2]. Although sophisticated conjectures about the ultimate nature of interatomic forces have a centuryold history [3], the first successful physical explanation, for instance, for the force between two polarizable, spherically symmetrical atoms, was derived only when a fully quantum mechanical treatment became possible (see review in Ref. [4] and references therein).

Once the origin of "centers of van der Waals attraction" [1] was understood, focus shifted to the computation of surface forces between boundaries of arbitrary shape, such as two infinite parallel planes [5] or two macroscopic spherical particles separated by a gap [6], which could either be empty or filled with another substance. By making the assumption of additivity, results were obtained relatively straightforwardly by a pairwise addition of van der Waals forces between pairs of atoms, each belonging to one of the two surfaces [2]. Despite the early successes of the London theory, discrepancies

\footnotetext{
*e-mail: fpinto@jazanu.edu.sa
}

between theoretical predictions and experimentation persisted and were eventually attributed to retardation, that is, the effect of finiteness of the speed of light, on the detailed dependence of dispersion forces on interatomic distance. Although it had already been suggested that retardation plays a critical role [2], it was Hendrik Casimir who first employed a zero-point-energy argument to treat the interaction of two perfect parallel plane reflectors separated by a gap, thus reproducing the observed more rapid decrease of the inter boundary force for relatively large gaps [7] (notice that, in common practice and unless otherwise specified, the terms Casimir and van der Waals forces are used interchangeably and without specific reference to the retardation regime).

However, the case of perfect reflectors is a very extreme idealization and practical applications required a treatment capable to deal with the interaction between real boundaries with given optical properties. This was accomplished by Lifshitz for two semi-infinite parallel slabs through extremely cumbersome calculations based on the introduction of Rytov's noise source into the field equations [8]. The Lifshitz theory of dispersion forces represents a landmark achievement as it allows for the treatment of real materials, it displays the transition from the unretarded to the retarded regimes, it removes the postulate of additivity, and it recovers all previously known results, such as those of London, Hamaker, and Casimir, in their appropriate limits [9]. Importantly, however, the Lifshitz theory could not be trivially extended beyond the archetypal case of two parallel slabs so that the study of realistic geometries [10], including surface roughness [11], 
had to wait for much more sophisticated analytical and numerical treatments in very recent times.

More broadly, it is now appreciated that Casimir effects are common to all fields, whether classical or quantum, once they are modified by the introduction of boundaries [12] even if those boundaries are not "hard" but "soft" [13]. Hence implications include proof of the contribution to hadron masses by the confined quark and gluon fields in the MIT bag model [14], experimental attractive and repulsive acoustic Casimir effects due to random noise [15], and the fascinating possibility to directly detect the gravitational equivalent of the atomic interaction energy in curved space-time by means of atomic traps [16, 17]. It is important to appreciate that entirely different formulations of the electrodynamical Casimir force between surfaces can be produced, which all remarkably lead to the same expression found by Lifshitz [12]. For instance, it is possible to attribute the dispersion force to modes existing within the gap between two slabs, or to regard the slabs themselves as the source of such interaction. Along these lines, London provided a model of dispersion forces between individual atoms based on interacting oscillators in perturbation theory [18]; however, a correct understanding of the Casimir force can be gained by concentrating on the effect of boundaries on the zero-point-energy of the electromagnetic field in the gap $[19,20]$. Such apparent lack of sensitivity of the results derived by Lifshitz on fundamental assumptions has led to ongoing intense research into the structure of the theory even with philosophical implications regarding the ultimate nature of the quantum vacuum [21].

Any verification of theoretical predictions requires the accurate experimental measurement of dispersion forces between surfaces at ranges well below $10 \mu \mathrm{m}$. In that regime, the pressure diverges very quickly and it can rise to $1 \mathrm{~atm}$ at distances of $\approx 10 \mathrm{~nm}$. The most successful strategy demonstrated on a macroscopic scale is that devised by Lamoreaux by means of a modified torsion pendulum [22, 23]. A completely different approach, based on the use of a modified atomic force microscope (AFM), was demonstrated and further developed by the group led by Mohideen and collaborators [24]. Casimir force experimentation presents various practical challenges both of implementation and interpretation and fierce debates have arisen around conflicting claims of accuracy and the ultimate meaning of the results produced by different groups [25].

Interestingly, the quantum field theoretic approach not only contributed a much more sophisticated understanding of the fundamental issues but it also injected an enduring perception of this class of phenomena as, in some sense, exotic. Hence the Casimir effect came to be described as "... one of the least intuitive consequences of quantum electrodynamics" [26] and variously characterized by leading researchers as, for instance, "small" [7], "tiny" [27], and "...too weak to command great attention" [28]. This must be contrasted with the remarkable anticipation of device stiction phenomena [29] by Feynman in 1959 and his amusing description of van der Waals forces as the cause of future challenges as in ". . . those old movies of a man with his hands full of molasses" [30]. Unexpectedly, especially considering the industrial origins of Casimir's problem recalled above, surprise by physicists at the dominance of dispersion forces on the nanoscale has been reported by the media well into the 21 st century [31]. In contrast, but simultaneously, realization of the industrial potential of van der Waals forces derived from the study of biological systems has led to the development of such novel products as "gecko glue" [32-35].

However, the suggestion that van der Waals forces not only represent a design limitation but should be considered as an enabling technological resource is not new. For instance, this idea was clearly articulated by physicist, inventor, and writer Robert L. Forward in 1984 [36]. His concept of a "vacuum-fluctuation battery," designed to achieve an exchange between electrical and van der Waals field energy through interacting thin foils, although impractical, highlighted some typical, enduring obstacles. This includes the need for extremely small interboundary separations and large specific interacting areas, and the problem of dynamical stability, which we further analyze below. This latter factor was explored in detail within the context of MEMS dynamics by Maclay and collaborators, who provided a first idealized model describing the departure of an oscillating micro-electro-mechanical system (MEMS) from its linear regime in which Casimir forces are present [37].

\section{Thermodynamical considerations}

Although the above developments clearly show that van der Waals forces may decisively affect nanodevice performance, the event that reshaped our understanding of their full technological potential was the discovery that such forces can be manipulated. Specifically, van der Waals forces, or the quantum vacuum closely connected to their existence, can serve as a "dynamic material", which can be addressed as needed in both space and time [38]. This property was first demonstrated in Germany by means of a relatively little cited but pivotal experiment carried out as a follow up to Siegfried Hunklinger's doctoral dissertation. In that initial effort, a condenser microphone was modified by attaching a small sensing dielectric disk at the center of its vibrating membrane. A spherical lens was then brought within micrometer range of the disk and driven into oscillatory motion. Since van der Waals forces are extremely sensitive to the gap width, the variable interboundary lens-disk force drove the microphone into vibration when placed in a moderate vacuum. This novel dynamic detection strategy confirmed theoretical expectations from the Lifshitz theory regarding forces between dielectrics [39-40].

As a later key improvement, the authors deposited a dot of amorphous silicon at the center of the spherical lens on the side facing the sensing disk. Upon back-illuminating such a deposition, the van der Waal 
force was observed to increase [41]. Qualitatively, such a change is expected because the dispersion force equation derived from the Lifshitz theory explicitly depends on the dielectric functions of the interacting materials, as well that of the material in the gap, if that is filled with a medium. Since illumination alters the charge carrier density in the amorphous silicon, and therefore its reflectivity, it is expected that the dispersion force shall be affected, as is indeed observed. Importantly, the results did not conform to all detailed expectations from the Lifshitz theory and research into this specific problem is ongoing in our Laboratory [42-43]. However, a different implementation of this experiment, by means of the AFM approach mentioned above, later claimed to have fully confirmed the theoretical predictions [44].

Although the technological implications of the experiments carried out by Hunklinger and collaborators were not fully understood for two decades, the present author first showed that van der Waals force modulation enables thermodynamical engine cycles to be executed on the nanoscale [45]. In other words, in analogy with a classical steam engine, pressure upon a "piston" can be altered upon energy exchanges between the van der Waals system and appropriate heat reservoirs. Of course, unlike the typical case, if the van der Waals force is attractive, transferring energy into (out of) the system from a high (low) temperature reservoir is followed by a piston downstroke (upstroke), corresponding to a clockwise cycle in a $(p, V)$ diagram of the semiconductor system above (we are neglecting the intrinsic dependence of the Casimir force on temperature predicted by the Lifshitz theory).

Research into the properties of such engine cycles has exposed two main lines of inquiry. Firstly, the present author has introduced a series of gedanken experiments to show that modeling van der Waals thermodynamical transformations, so as to satisfy the first law of thermodynamics, is not trivial. Although it is clear that transferring energy into the system generally results into changes of the dielectric functions which enter the Lifshitz theory, in order to achieve energy conservation over a closed cycle, the models must also include changes of the dielectric functions due to the interaction of the two boundaries. This is mandatory in order to ensure that the energy released by the system to decrease the van der Waals force before the upstroke be different than the energy transferred into the system before the downstroke and, at most, equal to the net mechanical work done by the system [45, 46].

In this context, it is critical to notice that the standard formulation of the Lifshitz theory does not include any dependence of the effective dielectric functions on the gap width. In fact, the dielectric functions typically used are those obtained from independent optical measurements, either via numerical interpolation of tabulated data or from analytical models and no dependence on the gap width is considered by the overwhelming majority of authors in the literature. It is now clear that this naïve approach leads to Casimir force engine cycles that cannot conserve energy. Hence the present thermodynamical approach allows one to constrain the behavior of the dispersion force by demanding energy conservation.

As we further discuss below, this is of timely importance since suggestions have already been made, on different considerations, that the Lifshitz theory may fail, even qualitatively, in excited matter and in systems out of equilibrium, such as is the case in dynamically illuminated semiconductors. For instance, according to such claims, it might be incorrect to calculate the Lifshitz force in excited matter simply from the dielectric function of the excited boundaries [47-55]. It is relevant to stress that research into related energy conservation implications of the Casimir effect and quantum vacuum theory is ongoing and it is extremely critical to identify limitations of some idealizations, further potential applications, and paradoxes [36, 45-46].

Also, it was experimentally proven that systems with rapidly moving boundaries, equivalent to boundaries with fast changing dielectric properties, can transform mechanical energy into real photons - the dynamical Casimir effect - thus affecting the evolution of the system via quantum friction, although the existence of this phenomenon has been surrounded by a recent fierce debate in the refereed literature [56-71].

The second, parallel line of enquiry focuses on the identification of systems in which technological performance can be drastically enhanced or entirely novel capabilities introduced by adopting van der Waals manipulation strategies and appropriate engine cycles. For instance, this has led to consideration of applications in adaptive optics, robotic nanoactuation, and nanotube array energy storage and propulsion, to cite just a few [72-74]. Although we offer some general considerations of wider applicability, the focus of the rest of this paper will be on investigating the above issues in systems in which the interboundary gap is well below $1 \mathrm{~nm}$, corresponding to the unretarded regime, and lateral dispersion forces are applied to a shuttle moving within nanotube walls (see below).

\section{Telescoping nanotube core dynamics}

A multi-walled nanotube, in which both end-caps have been removed so that its inner core is free to slide within the outer shells, is a remarkable example of a system dynamically dominated by van der Waals interactions. If the core is partially extruded, for instance mechanically, and then released, it is expected that many oscillations will be completed before it comes to rest under the action of relatively small frictional forces. Because of the extremely small interboundary distance (approximately $0.34 \mathrm{~nm}$ ) and thus large van der Waals pressures, and the small typical core mass, natural oscillation frequencies are in the $\sim 100 \mathrm{GHz}$ range, leading to the suggestion that such systems could be employed in next-generation ultra-high-frequency oscillators, sensors, and robotic tools [4]. The ultimate origin of this 
retraction force, as demonstrated from direct experimentation [75-76], is the unretarded van der Waals interaction energy between the outer nanotube walls and the embedded core surface, which, neglecting edge effects, is proportional to the first power of the core length within the nanotube (including short-range atomic repulsive interactions). Therefore, unsurprisingly, by taking the opposite of the first derivative of such energy with respect to the longitudinal position of the core, a constant force is obtained for an extruded core, and a vanishing force results for a completely embedded core (assuming the core is shorter than or at most equal to in length to the outer shells) [77-79]. It is important to stress that the energy, and not the force, is proportional to displacement so that the system is highly non-linear (TNL, or Truly NonLinear in Mickens' sense [80]) since no harmonic regime for small-oscillations exists.

Although nanotube oscillating cores appear to hold great promise in technological applications, several difficulties remain. Some pertain to synthesis, including identifying processes for the consistent removal of the caps so the inner core can be actuated [75-76]. Even more fundamental is the difficulty to set the completely embedded core into motion. This author has previously shown that this long standing problem can be successfully addressed, in principle, by establishing a dispersion force gradient along the nanotube axis, for instance by partial laser illumination of the outer walls [4]. Strictly connected to this challenge is the need to transfer energy to the core so as to maintain its forced oscillations, which are dampened by various friction forces. Furthermore, in order to carry out any useful technological task, the core must interact with the outside environment, for instance, by doing mechanical work as in a nanorobot, or producing an electric signal as in a nanosensor or nanobattery, or establishing electrical contact as in a nanoswitch or memory device [4]. These interactions introduce additional forces, such as the electrostatic forces between the partially extruded core cap and the outer nanotube walls [81], or the electrostatic and Casimir forces between the cap and nearby facing electrodes [82].

Typical systems used to measure the Casimir force, including the torsion balance [22], the AFM [24], and the modified microphone membrane [39-40], are also acted upon by an elastic restoring force, which gives rise to at least one position of stable equilibrium for the sensing element [82]. As the interboundary gap is decreased and the Casimir force correspondingly increases, all positions of equilibrium disappear [37] and the sensing element collapses towards the facing boundary - the so-called pull-in effect leading to device failure by stiction [83, 84]. On the contrary, given the details of the dependence on the core axial coordinate of the constant retracting force and all other forces, any position of static equilibrium in this system is typically unstable [85]. For instance, in an energy storage system in which energy is exchanged between the nanotube van der Waals field and a nanotubeelectrode electrostatic field, the core either remains inside the outer walls or immediately collapses onto the electrode. This limitation was recognized early on by Forward, who suggested the use of a feedback system to manage the process of collapse of interacting foils and the release of electrical energy in his idealized "battery" [36]. Similar concepts have been again proposed recently to stabilize telescoping nanotube accelerometers [86], although this intrinsic instability has also been exploited in studies of telescoping nanotube memory devices [87]. The present author has shown that stable positions of equilibrium can be introduced by dividing the nanotube into just two segments with slightly different outer wallcore van der Waals interaction energy. For instance, in an energy storage system, this strategy allows the user to address the position of the core by imposing an appropriate time-profile for the potential difference between the nanotube and the electrode, leading to a completely controlled charge-discharge process unavailable in electrochemical batteries or supercapacitors [85].

\section{Future directions}

In this section, we explore the manner in which studies of telescoping nanotube systems of the type described above can be expected to address fundamental issues relating to dispersion force physics and also lead to industrial applications. From the experimental standpoint, novel measurements designed to directly explore the regimes relevant to novel applications are indispensable to move "beyond gedanken experiments" [81]. A very important development has been the experimental confirmation of the possibility to use radiation to modulate nanotube spectra [88-89] as theoretically shown by this author from full 2-dimensional exciton screening models [90].

The core retraction force has also been directly measured in several experiments of great importance not only in applications but also in principle [91-93]. From this latter standpoint, the existence and behavior of this dominant interaction is a conclusive demonstration of the existence of lateral Casimir forces, that is, dispersion forces due to a dependence of the energy on the net overlapping area but at constant gap width, s. The details of the dependence of this force on all relevant physical parameters, including its typical proportionality $1 / s^{2}$, are consistently confirmed not only from the Lifshitz theory as seen above (with appropriate corrections $\sim 30 \%$ to the proportionality constant due to short-range atomic repulsive interactions [94]), but also from molecular dynamics simulations via direct pair-wise summation and in continuum approximations [75-79, 95] (see also Ref. 4, Sec. III-C and references therein).

The extreme speed of progress in this field, and the need to address an enduring "disjunction among disciplines" ([96], see p. 349) are clearly shown by the assessment that "[e]xperimental verification of lateral forces in flat plates is challenging but may be possible" [46], still being rendered a decade after the remarkable experiments by Zettl and collaborators [75] (notice that the 
effect of curvature on the Casimir energy of two coaxial cylinders is corrected by an application of the proximity force theorem, thus reducing the problem to that of two flat plates [97]).

The above results set the stage for studies of the dependence of van der Waals forces in nanotubes on illumination and temperature on very short timescales. A critical milestone will be the detection in telescoping nanotubes of effects analogous to those discovered in amorphous silicon depositions by means of modified microphone experiments [41]. In this context, it is important to stress that, although both the earlier microphone-based and the later AFM experiments have shown a dependence of van der Waals forces on illumination, neither attempted to employ such effects to achieve actuation, a goal yet to be demonstrated on any scale [43].

From the application standpoint, future studies will determine whether partial illumination schemes [98] or other strategies to break the axial symmetry of the interlayer surface cohesive energy density in nanotubes can be employed to actuate cores initially at rest while wholly embedded within the outer walls. A landmark result will be the demonstration of dispersion force-driven oscillators and accelerators, since systems of this type would provide the tools necessary to explore high speed motion and frictional sources in nanotube shells. Hence nanotube core accelerators [4] could be employed as remarkable novel laboratories for the study of dispersion forces in both carbon and non-carbon nanotubes, thus contributing to discriminate amongst competing quantum electrodynamics theories in excited matter and possibly unambiguously revealing quantum friction. Such nanodevices could be arbitrarily shaped and even explore high-energy fullerene-fullerene head-on collisions, thought to play an important part in the fusion and coalescence of fullerene cages [99]. In perspective, experiments on accelerators and oscillators of this type will quantitatively answer fundamental questions regarding energy conservation in Casimir force theory.

From the mathematical standpoint, a decisive leap in sophistication must be achieved to describe dispersion force manipulation in nanotubes. As is also the case in bulk materials [47], illumination demands a specific treatment of carrier diffusion processes in lower dimensional structures [98]. So far, dynamical analyses of the response of driven nanotube cores, conducted both in the continuum approximation and by means of molecular simulations [4], have naively posited an external exciting force, for instance with a sinusoidal behavior [87, 100101]. Such initial studies have exposed the challenge of controlling an extremely complex behavior consistent with the truly-non-linear (TNL) dynamics of these systems. However, unlike what occurs, for instance, in the linear regime of MEMS cantilever oscillations, no proposed actuation mechanism can be even ideally described as a simple driving harmonic term. Many aspects of nanotube physics become simultaneously engaged in dispersion force modulation strategies on the nanoscale, such as the time dependent evolution and diffusion of charge carrier density, thermal properties, spectra, friction, multi-dimensional mechanical vibrations, and effects of external coupling forces. Given the extreme sensitivity of truly non-linear systems to initial conditions and external excitation, next-generation, computation intensive models will be necessary to determine whether any previously drawn conclusions survive in such much more realistic models. In this context, it must be stressed that a vigorous debate is unfolding regarding the validity of existing dispersion force theories away from the idealizations of the Lifshitz theory.

It is clear that technological applications can only emerge and prevail in the market place if nanotube core actuation can be transformed from a highly non-linear process to one endowed with predictable islands of stability. In this sense, "sculpting" the dispersion force field experienced by the oscillating element will provide a critical design strategy as already shown by this author in the case of storage systems [85]. This approach will be extended to much more sophisticated and complex implementations both in space and time.

The technological promise of dispersion force manipulation-driven nanotube core actuation, oscillation, and high speed expulsion encompasses a remarkable spectrum of areas of application, ranging from robotic nanosurgery deep inside the human body to high energy density storage with hyperfast recharge and discharge, from ultra-high oscillators in next generation communication systems to high-thrust high-endurance space propulsion systems. The path to reduce all such concepts to practice is being clarified and exciting developments in theory, experimentation, industry, and product development will certainly result within a reasonable time horizon.

\section{Acknowledgments}

Partial funding under a Jazan University Deanship of Scientific Research grant and the Jazan University Department of Physics is gratefully acknowledged. Nurdogan Can (Jazan University) provided additional indispensable logistical support and continued encouragement. Thanks are due to the organizers of the Saudi International Meeting on Frontiers of Physics (SIMFP) series held annually at Jazan University, the Material Science \& Engineering Program of the King Abdullah University of Science and Technology (KAUST), the Center of Excellence in Nanotechnology (CENT) and King Fahd University of Petroleum and Minerals (KFUPM) physics department, and the Atomic, Molecular, and Optical Physics section of the Saudi Physical Society (AMOPSPS), for several invitations to present selections of the material discussed in this review. Prof. Ahmet Yavuz Oral (Gebze Technical University) kindly hosted an invited talk on the subject of this paper at the " 5 th International Advances in Applied Physics \& Materials Science Congress \& Exhibition" (APMAS2015) held in FethiyeMuğla, Turkey. 


\section{References}

[1] F. London, J. Phys. Chem. 46, 305 (1942).

[2] E.J.W. Verwey, J.Th.G. Overbeek, Theory of the Stability of Lyophobic Colloids, Ch. 6., Elsevier, New York, 1948.

[3] J.S. Rowlinson, Cohesion - A Scientific History of Intermolecular Forces, Cambridge University Press, Cambridge 2002.

[4] F. Pinto, 48th AIAA/ASME/SAE/ASEE Joint Propulsion Conference \& Exhibit, AIAA 2012, p. 3713.

[5] J.H. de Boer, Trans. Faraday Soc. 32, 10 (1936).

[6] H. Hamaker, Physica 4, 1058 (1937).

[7] H.B.G. Casimir, Proc. Kon. Ned. Akad. Wetenshap 51, 793 (1948).

[8] E.M. Lifshitz, Sov. Phys. JETP. 2, 73 (1956).

[9] I.E. Dzyaloshinskii, E.M. Lifshitz, L.P. Pitaevskii, Adv. Phys. 10, 165 (1961).

[10] F. Pinto, Phys. Rev. A 80, 042113 (2009).

[11] P.J. van Zwol, G. Palasantzas, Acta Phys. Pol. A 117, 379 (2010).

[12] P.W. Milonni, The Quantum Vacuum, Academic Press, San Diego, 1994.

[13] A.A. Actor, I. Bender, Phys. Rev. D 52, 3581 (1995).

[14] K. Johnson, Acta Phys. Pol. B 6, 865 (1975).

[15] A.S. Larraza, Ch.D. Holmes, R.T. Susbilla, B. Denardo, J. Acoust. Soc. Am. 103, 2267 (1998).

[16] F. Pinto, Phys. Rev. D 73, 104020 (2006).

[17] F. Pinto, Int. J. Mod. Phys. D. 15, 2235 (2006)

[18] F. London, Trans. Faraday Soc. 33, 8 (1937).

[19] E.A. Power, Introductory Quantum Electrodynamics, American Elsevier, New York 1965.

[20] D.L. Andrews, L.C. Dávila Romero, Eur. J. Phys. 22, 447 (2001).

[21] W.M.R. Simpson, Stud. Hist. Philos. M.P. 48, 84 (2014).

[22] S.K. Lamoreaux, Phys. Rev. Lett. 78, 5 (1997).

[23] S.K. Lamoreaux, Phys. Rev. A 82, 024102 (2010).

[24] U. Mohideen, A. Roy, Phys. Rev. Lett. 81, 4549 (1998).

[25] S.K. Lamoreaux, Annu. Rev. Nucl. Part. Sci. 62 , 37 (2012).

[26] J. Schwinger, L.L. DeRaad, K.A. Milton, Ann. Phys. (N.Y.) 115, 1 (1978)

[27] P.W. Milonni, Contemp. Phys. 33, 313 (1992).

[28] D. Kleppner, Phys. Today 43, 9 (1990).

[29] E. Buks, M.L. Roukes, Phys. Rev. B 63, 033402 (2001).

[30] R.P. Feynman, Engineering and Science 23, 22 (1960).

[31] K. Chang, "A Tiny Force of Nature is Stronger than Thought", New York Times, February 9th, 2001, p. A17; see also "Much Ado About Nothing", The Economist, May 24th-30th, 2008, p. 105.
[32] K. Autumn, Y.A. Liang, S. Tonia Hsieh, W. Zesch, W. Pang Chan, T.W. Kenny, R. Fearing, R.J. Full, Nature 405, 681 (2000).

[33] A. Mahdavi, L. Ferreira, C. Sundback, J.W. Nichol, E.P. Chan, D.J.D. Carter, C.J. Bettinger, S. Patanavanich, L. Chignozha, E. Ben-Joseph, A. Galakatos, H. Pryor, I. Pomerantseva, P.T. Masiakos, W. Faquin, A. Zumbuehl, S. Hong, J. Borenstein, J. Vacanti, R. Langer, J.M. Karp, PNAS 105, 2307 (2008).

[34] J.M.R. Bullock, W. Federle, Naturwissenschaften 98 , 381 (2011).

[35] K. Autumn, N. Gravish, Phil. Trans. R. Soc. A 366 1575 (2008).

[36] R.L. Forward, Phys. Rev. B 30, 1700 (1984).

[37] F.M. Serry, D. Walliser, G.J. Maclay, J. Microelectromech. Sys. 4, 193 (1995).

[38] F. Pinto, Am. Sci. 102, 280 (2014)

[39] S. Hunklinger, Ph.D. Thesis (unpublished), T.H. München, 1969.

[40] S. Hunklinger, H. Geisselmann, W. Arnold, Rev. Sci. Instrum. 43, 584 (1972).

[41] W. Arnold, S. Hunklinger, K. Dransfeld, Phys. Rev. B 19, 6049 (1979).

[42] F. Pinto, J. Sound. Vib. 291, 1278 (2006).

[43] F. Pinto, J. Phys. A 41, 164033 (2008).

[44] F. Chen, G.L. Klimchitskaya, V.M. Mostepanenko, U. Mohideen, Opt. Exp. 15, 4823 (2007).

[45] F. Pinto, Phys. Rev. B 60, 14740 (1999).

[46] G.J. Maclay, Phys. Rev. A 82, 032106 (2010).

[47] N. Inui, J. Phys. Soc. Japan 73, 332 (2004).

[48] S.Y. Buhmann, L. Knoll, D.-G. Welsch, H.T. Dung, Phys. Rev. A 70, 052117 (2004).

[49] P.R. Berman, G.W. Ford, P.W. Milonni, Phys. Rev. A 89, 022127 (2014).

[50] Y.B. Sherkunov, Phys. Rev. A 72, 052703 (2005).

[51] F.S.S. Rosa, D.A.R. Dalvit, P.W. Milonni, Phys. Rev. A 81, 033812 (2010).

[52] T.G. Philbin, New J. Phys. 12, 123008 (2010).

[53] T.G. Philbin, New J. Phys. 13, 063026 (2011).

[54] S.A.R. Horsley, Phys. Rev. A 86, 023830 (2012).

[55] V.E. Mkrtchian, C. Henkel, Ann. Phys. 526, 87 (2014).

[56] E.V. Teodorovich, Proc. R. Soc. Lond. A 362, 71 (1978).

[57] J. Mahanti, J. Phys. B: Atom. Molec. Phys. 13, 4391 (1980)

[58] W.L. Schaich, J. Harris, J. Phys. F: Metal Phys. 11, 65 (1981).

[59] L.S. Levitov, Europhys. Lett. 8, 499 (1989).

[60] J.B. Pendry, J. Phys.: Condens. Matter 9, 10301 (1997).

[61] M. Kardar, R. Golestanian, Rev. Mod. Phys. 71, 1233 (1999).

[62] A.A. Kyasov, G.V. Dedkov, Surf. Sci. 463, 11 (2000).

[63] T.G. Philbin, U. Leonhardt, New J. Phys. 11 , 033035 (2009). 
[64] J.B. Pendry, New J. Phys 12, 033028 (2010).

[65] U. Leonhardt, New J. Phys. 12, 068001 (2010).

[66] J.B. Pendry, New J. Phys 12, 068002 (2010).

[67] K.A. Milton, Am. J. Phys. 79, 697 (2011).

[68] A.I. Volokitin, B.N.J. Persson, New J. Phys 13, 068001 (2011).

[69] M.F. Maghrebi, R. Golestanian, M. Kardar, Phys. Rev. A 88, 042509 (2013).

[70] J.S. Høye, I. Brevik, Eur. Phys. J. D 68, 61 (2014).

[71] M.G. Silveirinha, New J. Phys. 16, 063011 (2014).

[72] F. Pinto, in: Optomechatronic Technologies 2008, Eds. Y. Otani, Y. Bellouard, J.T. Wen, D. Hodko, Y. Katagiri S.K. Kassegne, J. Kofman, S. Kaneko, C.A. Perez, D. Coquin, O. Kaynak, Y. Cho, T. Fukuda, J. Yi, F. Janabi-Sharifi, SPIE, San Diego, 2008, p. 726616.

[73] F. Pinto, in: Space Technology and Applications International Forum (STAIF-2008), AIP Conference Proceedings 969, Ed. M.S. El-Genk, American Institute of Physics, Melville, NY 2008, p. 959.

[74] F. Pinto, US Patent Nos. 8,299,761; 8,174,706; $8,149,422 ; 6,920,032 ; 6,842,326 ; 6,665,167 ; 6,661,576$ $6,650,527 ; 6,593,566 ; 6,477,028$.

[75] J. Cumings, A. Zettl, Science 289, 602 (2000).

[76] A. Kis, A. Zettl, Phil. Trans. R. Soc. A 366, 1591 (2008).

[77] W. Guo, Y. Guo, H. Gao, Q. Zheng, W. Zhong, Phys. Rev. Lett. 91, 125501 (2003).

[78] D. Baowan, J.M. Hill, Z. angew. Math. Phys. 58 857 (2007).

[79] V. Zavalniuk, S. Marchenko, Low Temp. Phys. 37, 337 (2011).

[80] R.E. Mickens, Truly Nonlinear Oscillations, World Scientific, New Jersey, 2010.

[81] L. Forro, Science 289, 560 (2000).

[82] A.W. Rodriguez, F. Capasso, S.G. Johnson, Nature Photonics 5, 211 (2011).

[83] E. Buks, M.L. Roukes, Europhys. Lett. 54, 220 (2001).
[84] X.L. Jia, J. Yang, S. Kitipornchai, Acta Mech. 218, 161 (2010).

[85] F. Pinto, in: Nanotube Superfiber Materials, Ch. 27, Eds. M. Schulz, V.N. Shanov, Y. Zhangzhang, Elsevier, New York, 2013.

[86] J.W. Kang, J.H. Lee, K.-S. Kim, Y.G. Choi, Model. Simul. Mater. Sci. Eng. 17, 025011 (2009).

[87] J.W. Kang, Q. Jiang, Nanotechnology 18, 095705 (2007).

[88] C.L. Yang, B. Hou, I.L. Li, Z.M. Li, Z.K. Tang, J.N. Wang, H.J. Liu, W.K. Ge, Phys. Rev. B 71, 233404 (2005).

[89] D. Rossouw, M. Bugnet, G.A. Botton, Phys. Rev. B 87, 125403 (2013).

[90] F. Pinto, J. Appl. Phys. 114, 024310 (2013).

[91] S. Akita, Y. Nakayama, Jpn. J. Appl. Phys. 42 , 4830 (2003).

[92] B.H. Hong, J.P. Small, M.S. Purewal, A. Mullokandov, M.Y. Sfeir, F. Wang, J.Y. Lee, T.F. Heinz, L.E. Brus, P. Kim, K.S. Kim, PNAS 102, 14155 (2005).

[93] A. Kis, K. Jensen, S. Aloni, W. Mickelson, A. Zettl, Phys. Rev. Lett. 97, 025501 (2006).

[94] H. Krupp, Advan. Colloid Interface Sci. 1, 111 (1967).

[95] Q. Zheng, Q. Jiang, Phys. Rev. Lett. 88, 045503 (2002).

[96] E.V. Blagov, G.L. Klimchitskaya, V.M. Mostepanenko, Phys. Rev. B 75, 235413 (2007).

[97] V.A. Parsegian, Van der Waals Forces, Cambridge Univ. Press, Cambridge, 2006.

[98] A. Achoyan, S. Petrosyan, H. Ruda, A. Shik, Phys. Rev. B 77, 085303 (2008).

[99] B. Adhikari, Phys. Stat. Sol. A 211, 277 (2014).

[100] A. Neild, T.W. Ng, Q. Zheng, EPL 87, 16002 (2009).

[101] I. Kovacic, J. Sound. Vib. 330, 4313 (2011). 